\title{
A SIMO Hybrid Visible Light Communication System for Optical IoT
}

\author{
Shivani Rajendra Teli, Petr Chvojka, Stanislav Vitek, Stanislav Zvanovec (Senior Member, IEEE), \\ Rafael Perez-Jimenez, and Zabih Ghassemlooy (Senior Member, IEEE)
}

\begin{abstract}
Visible light communications (VLC) is an emerging technology utilizing light emitting diodes and photodetectors (PDs) and cameras (i.e., image sensors (ISs)) as the transmitter and receivers (Rx), respectively for simultaneous data communications, illumination, localization and sensing in indoor optical Internet of Things. We propose, a single-input multipleoutput (SIMO) hybrid VLC system using PD- and IS-based Rxs for simultaneous high- and low-speed (i.e., $\boldsymbol{R}_{b \text {-High }}$ and $\boldsymbol{R}_{b \text {-Low) data }}$ transmission. In addition, we propose amplitude overlapping $A_{\mathrm{OL}}$ in the modulation format to increase the $R_{b}$ and reduce the attenuation due to high-pass filter effect of the bias-T. We experimentally evaluate the performance of the proposed scheme considering the inter-link impacts. Results show that, at $A_{\mathrm{OL}}$ of 0 0.2 , (i) the measured bit error rate for the PD-based VLC is below the forward error correction limit of $3.8 \times 10^{-3}$ for $R_{b \text {-Low }}$ of 2.5 and

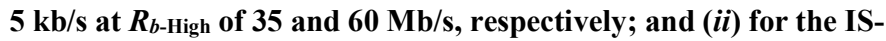
based VLC link with the camera gain of $4 \mathrm{~dB}$ and $R_{b}$-High of up to $70 \mathrm{Mb} / \mathrm{s}$, the reception success rates are $>96$ and $90 \%$ at $\boldsymbol{R}_{b \text {-Low } \text { of }}$ 2.5 and $5 \mathrm{~kb} / \mathrm{s}$, respectively.
\end{abstract}

Index Terms - Internet of Things (IoT), single-input multiple output (SIMO), light emitting diodes (LEDs), visible light communications (VLC), photodiode (PD), optical camera communications (OCC), camera, image processing.

\section{INTRODUCTION}

$\mathrm{R}$ ECENT developments in Internet of Things (IoT) stems largely from interconnections and communications between a number of users and a plethora of different devices, collectively referred to as the smart devices connected to the wireless cellular networks [1]. This wireless-based IoT assists in developing and implementing the smart indoor environments (i.e., homes, offices, hospitals and industries), which enable interactions between smart devices and humans, as part of the fifth and sixth generation wireless networks. The major requirements in IoT-based communications system designs are [2]: (i) human value - smart applications, substantial benefits, quality of life and better utilization of resources; ( $i i)$ data value - secured links and fast processing; (iii) connectivity - protocol developments; and (iv) efficiency and availability. Note that, it is challenging to fulfil the aforementioned requirements by only relying on the costly and frequency spectrum congested radio frequency (RF) technologies. Alternatively, in many applications, where RF cannot be deployed due security

This work was supported by the European Union's Horizon 2020 Research and Innovation Programme, under the Marie Sklodowska-Curie grant agreement no 764461 (VisIoN). (Corresponding author: S. R. Teli.)

S. R. Teli, P. Chvojka, S. Vitek, and S. Zvanovec are with the Faculty of Electrical Engineering, Czech Technical University in Prague, Technicka, 16627 Prague, Czech Republic (email: telishiv@fel.cvut.cz; chvojka.petr@gmail.com; viteks@fel.cvut.cz; xzvanove@fel.cvut.cz). reasons, insufficient spectrum, etc., the optical wireless communications (OWC) technology covering ultraviolet, infrared, and visible bands can be adopted in IoT as part of the $5 \mathrm{G}$ wireless networks [3]. OWC utilizing the visible light spectrum (i.e., $\sim 370-780$, nm), known as visible light communications (VLC) with a useable bandwidth $B$ of $\sim 400$ $\mathrm{THz}$, which is 10,000 times wider than RF $[4,5]$, uses lightemitting diodes (LEDs) luminaries and photodetectors (PDs) to simultaneously provide data communications, illumination, localization, and sensing in mostly indoor environments [4]. VLC systems with data rates $R_{\mathrm{b}}$ within the range of $100 \mathrm{Mb} / \mathrm{s}$ to $15.7 \mathrm{~Gb} / \mathrm{s}$ using polarization division multiplexing [6] and orthogonal frequency division multiplexing (OFDM) with adaptive bit loading technique [7 - 9], respectively have been reported in the literature.

Utilizing LED-based lighting infrastructures offer benefits such as lower power consumption, longer life expectancy, lower flicker factor, lower heat generation, and fast switching, where the latter is highly desirable in high-speed applications $[4,5]$. To this end, extensive works have been reported by extending the point-to-point VLC links defined in IEEE 802.15.7 standard [10] also termed as light-fidelity (LiFi). LiFi utilizing off-the-shelf LEDs was proposed as an enabler for the IoT in indoor environments [11] with three main features such as precise positioning, power delivery since energy can be harvested from light, and inherent security [11]. In [12], the LiFi-enabled bidirectional IoT communications system with visible and infrared lights used in the downlink and uplink, respectively was investigated. In the proposed bidirectional LiFi-IoT system, a non-orthogonal multiple access scheme with the quality-of-service guaranteed optimal power allocation strategy was adopted to maximize the energy efficiency of both down- and up-link [12]. Therefore, the realization of all optical IoT (OIoT) using the VLC/OCC technology with multiuser bidirectional transmission [13], multiple access [14], and handover [15] features has been made possible [16].

Hybrid OWC schemes using fiber links to transmit highspeed signals from the base station/optical line terminal to the indoor access network/optical network unit, and VLC-PD links in an indoor access network have been proposed in the literature. In [17], M-ary amplitude shift keying (MASK)

R. Perez-Jimenez is with the Institute for Technological Development and Innovation in Communications, Universidad de Las Palmas de Gran Canaria, 35001 Las Palmas, Spain (email: rperez@idetic.eu).

Z. Ghassemlooy is with the Optical Communications Research Group, Northumbria University, Newcastle-upon-Tyne NE1 7RU, UK (email: z.ghassemlooy@northumbria.ac.uk). 
quadrature amplitude modulation (QAM)-OFDM using a 2 $\mathrm{GHz}$ direct-modulated-laser (DML) with net data rates up to 4.18 and $3.80 \mathrm{~Gb} / \mathrm{s}$ for fast Fourier transform sizes of 128 and 64 was reported. In [17], a lower frequency MASK signal modulates the power of each OFDM symbol, where the total power of an OFDM symbol denotes the MASK signal. The positive half and the full modulation depths were used to represent the low bit 0 and high bit 1, respectively. In [18], OFDM-based passive optical network was proposed for the hybrid wired and wireless optical access network. In the adaptive envelope modulation, multiple consecutive unipolar (CE) OFDM symbols are grouped together via symbols grouping and the power of each constant envelope CE-OFDM symbol group is determined by the M-ary pulse amplitude modulation (M-PAM) encoded wireless input data via power allocation. For indoor OWC, a DC bias is first added to the MPAM signal prior to intensity modulation of the LED. Following indoor free-space transmission, at the receiver the light focused on the PD via a lens. The regenerated electrical M-PAM signal is A/D converted and demodulated to generate the transmitted data stream.

On the other hand, VLC systems employing a IS-based receiver (Rx) better known as optical camera communications (OCC) (defined within IEEE 802.15.7 SG 7 [19]) have been gaining attention in the research community and industry because of the availability of smart devices integrated with high-speed and high-quality cameras [20]. Note, the CMOSbased cameras can capture images or record video streams using the global shutter (GS)- and rolling shutter (RS)-based capturing modes at different resolutions. The RS-based cameras can simultaneously capture multiple LEDs (i.e., in $\mathrm{ON}$ and OFF states) in a single frame, as rows of the image pixels are exposed to the light one at a time [21], thus achieving flickerfree transmission with increased $R_{b}$. However, $R_{b}$ is rather low mainly limited mainly by the camera frame-rate $f_{R}$. For human, $f_{R}$ is $16 \mathrm{fps}$, as this is the threshold to which the mind no longer perceives sequences as flashing images. For films, typically $f_{R}$ is used in the range of 24 to $48 \mathrm{fps}$, a value slightly above the perceptual limit, while in advanced smartphones with slow motion capabilities, $f_{R}$ could be as high as 120 fps with highdefinition formats such as $1280 \times 720$ pixels. For sport action and professional high-speed cameras $f_{R}$ are 240 and $>1000 \mathrm{fps}$, respectively.

Unlike the hybrid wired and wireless schemes proposed in $[17,18]$, the VLC system with hybrid PD- and camera-based Rxs can be used in short-range indoor OIoT applications for positioning, localization, navigation [22, 23], device-to-device communications (where higher $R_{b}$ is not the main requirement), mobile transactions, motion-based device control [24], small identification information, communications through advertisements [25], etc., which offer good degrees of mobility in the OIoT environments [24, 26]. A number of VLC systems for use in IoT have been reported including (i) DIMLOC dimmable LEDs in smart buildings using a smartphone camerabased Rx [22]; (ii) integrated VLC and VLC positioning networks [27]; (iii) Foglight - spatial encoding using a projector-based on the gray-coded binary images [23]; and (iv)
LiFi4IoT - that provides three individual motes such as $(a)$ retroreflector using downlink OCC and a liquid crystal shutter on top of a retroreflector, which enables the use of light backscattering as an uplink; (b) PD+LED operating in the sleep mode to harvest the energy from the ambient light for powering the IoT mote and for the up- and down-link; and (c) hybrid $\mathrm{RF} / \mathrm{LiFi}$ where the LiFi downlink and RF uplink are established [11]. Therefore, integrating both VLC-PD and OCC (IS-based VLC) links simultaneously can provide a versatile OIoT environment, where users can have a choice based on the device to switch between high- and low-speed VLC with PDs- and ISsbased Rx, respectively as shown in Fig. 1.

However, there are still challenges in the integration of VLC technology with hybrid PD- and camera-based Rxs in the OIoT environments, such as $(i)$ lower and limited $f_{R}$ in contrast to high-speed PDs; (ii) incompatibility in $R_{b}$ between VLC with PDs and IS, i.e., $R_{b \text {-High }} \gg R_{b \text {-Low }}$; (iii) the use of LEDs with different modulation bandwidth $B_{\mathrm{LED}}$ in PD- and IS-based VLC links, which can result in interference and noise in other's Rxs; and $(v)$ the increase in the overall implementation cost due to use of multiple LEDs. As a solution to these challenges, in this work, we propose a single-input multiple-output (SIMO) hybrid VLC system utilizing a single LED-based Tx, and PD- and ISbased Rxs for high- and low-speed data transmission simultaneously, respectively, see Fig. 1. Note, the high-speed PD-based VLC is mainly utilized for downloading big data, internet surfing, and online streaming, while the low-speed ISbased VLC is employed for transmission of short messages, identification, promotion information, control signals, and indoor localization information. It is envisaged that, the proposed scheme can provide versatile indoor services that allow users to receive data regardless of the devices used. For this reason, we propose a hybrid modulation format that can be used for both high- and low-speed VLC links, and have developed an experimental test-bed for verification. We present results for evaluating the link performance in terms of the bit error rate (BER) and the reception success by considering the impact of the low-speed IS-based VLC on the PD-based VLC and vice versa.

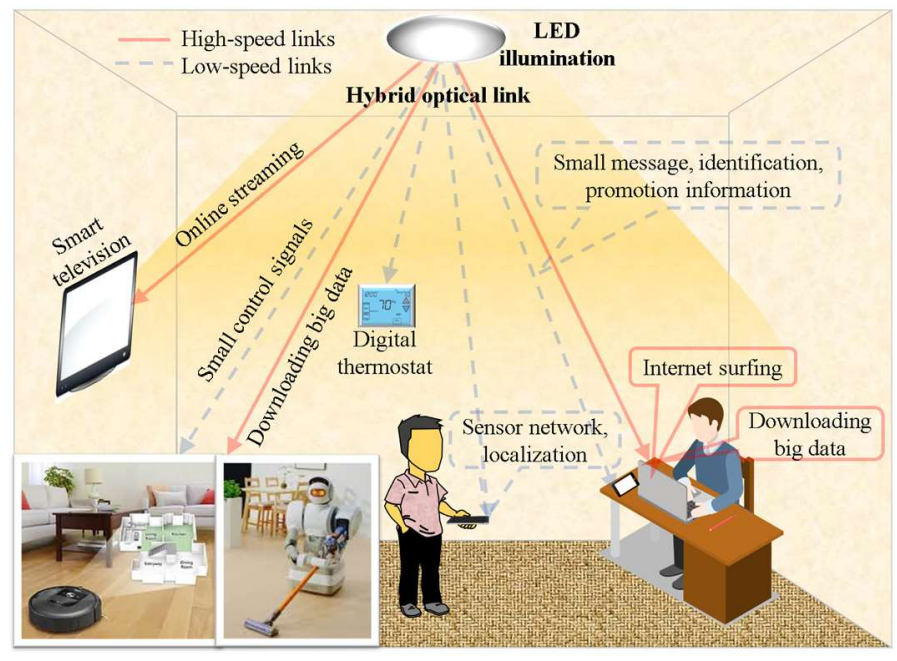

Fig. 1. SIMO hybrid VLC scheme: Concept of simultaneous high- and lowspeed data transmission for versatile OIoT based indoor environment.

The remaining sections are organized as follows: Section II 
gives a brief outlook and limitations of previous related works; Section III provides an overview of the proposed OIoT: SIMO hybrid VLC scheme; Section IV presents experiment results and discussion; Section V summarizes the performance and results of the proposed scheme and Section VI concludes the article along with future outlook.

\section{RELATED WORKS}

A. Fuzzy logic-based network selection in hybrid OCC/LiFi: A switching mechanism was used to select OCC or LiFi link (not both) based on the network selection factor obtained using the fuzzy logic [28]. Even though the major focus was on the network selection, the main points of modulation/demodulation, data processing, and BER performance for both links needs further investigation. The work was further extended using the fuzzy logic and roundrobin scheduling for assigning an appropriate network to the users in a hybrid OCC/LiFi system [29]. However, similar to the scheme in [28], the paper lacked details on simultaneous data transmission and processing.

B. VLC-OCC hybrid wireless systems: A 15 -Wt LED lamp is used for simultaneous data transmission at high- and low-speed in VLC and OCC link, respectively [30]. Note, to utilize the dimming functionality of LED, Manchester coding and variable pulse position modulation were used for OCC and VLC, respectively, where the later was transmitted only during 'high' duration of OCC (i.e., the ON state). However, the bandwidth efficiency of the system was low since only the ON state of the signal in the VLC link was utilized.

C. Hybrid OCC/LiFi with multilevel LED: Similar to [17, 18], a unipolar multilevel LED-based hybrid encoding scheme was proposed in [31]. In OCC and $\mathrm{LiFi}$, the data were transmitted using different power levels of the LED (i.e., intensity shift keying (ISK)) and multilevel OOK, respectively. In ISK, the 'high' and 'low' levels (i.e., bits ' 1 ' and ' 0 ') were modulated at power levels of $39,35.0$, and $36.7 \mathrm{dBm}$, respectively. However, to detect a small difference of $\sim 1 \mathrm{~dB}$ between high and low levels, a highly sensitive IS-based Rx with a highly precise and complex data processing is required. Moreover, changing the power levels corresponding to the varying amplitude depths of the ON and OFF bits, which affects signal detection and processing, will need further investigation.

The limitation of bandwidth efficiency and the effect of multilevel LED transmission based on varying amplitude depths of the ON and OFF signal levels in hybrid signal detection as well as the comprehensive analysis of data processing lacked in [28-31] are addressed in the proposed SIMO hybrid VLC scheme for OIoT.

\section{OPTICAL IOT: SIMO HYBRID VLC SCHEME}

As previously mentioned, unlike conventional PD-based VLCs with $R_{b}$ of $100 \mathrm{Mb} / \mathrm{s}$ up to $15.7 \mathrm{~Gb} / \mathrm{s}$ [6 - 9], the camerabased Rx in OCC has a limited $R_{b}$ of few $\mathrm{kb} / \mathrm{s}$ due to low capture speed of the camera i.e., ranging typically from 30 to $60 \mathrm{fps}$ [32]. As outlined above, there are several challenges that need addressing prior to full utilization of hybrid VLC using a single LED Tx for both PD- and IS-based VLC. The major advantage of this scheme is that, being able to use either links depending on the needs in indoor OIoT environments with reduced complexity. To demonstrate the SIMO hybrid VLC concept, we propose a bipolar modulation format that facilitates both lowand high-speed transmissions using a single LED, which is outlined below. This is followed by a detailed overview of the proposed SIMO hybrid VLC scheme.

\section{A. The Proposed Modulation Format}

Figure 2 shows the time waveforms of the proposed modulation format adopted in SIMO hybrid VLC, where the signal with $R_{b \text {-High }}\left(\right.$ i.e., with the bit duration $T_{b \text {-High }}$ shown in blue) is superimposed on the signal with $R_{b \text {-Low }}$ (i.e., $T_{b \text {-Low }}$ in orange). The data and the superimposed $s(t)$ signals are in the non-return-to-zero on-off keying (NRZ-OOK) and bipolar

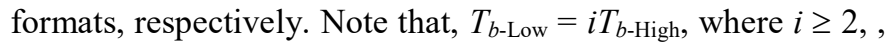
i.e., $R_{b \text {-High }}=i \cdot R_{b \text {-Low. }}$. Since $R_{b \text {-Low }}$ is the lower bound, then the LED is intensity modulated at the modulation frequency, which is given as:

$$
f_{s-\text { Low }}=R_{b-\text { Low }} \times N_{\text {Low }},
$$

where $N_{\text {Low }}$ is the number of samples of $R_{b \text {-Low }}$, which is given in terms of the number of samples of high-speed link $N_{\mathrm{High}}$ as:

$$
N_{\text {Low }}=\frac{R_{b-\mathrm{High}} \times N_{\mathrm{High}}}{R_{b-\text { Low }}}=\frac{f_{s-\mathrm{High}}}{R_{b-\text { Low }}} .
$$

From (2) and (1), we have:

$$
f_{s-\text { High }}=R_{b-\text { Low }} \times N_{\text {Low }}=f_{s-\text { Low }}
$$

Therefore, the generated signal $s(t)$ will contain two different

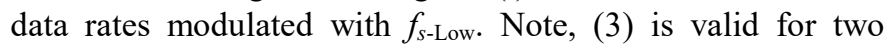
different data rates (i.e., $R_{b \text {-High }} \neq R_{b \text {-Low }}$ ), which is defined as:

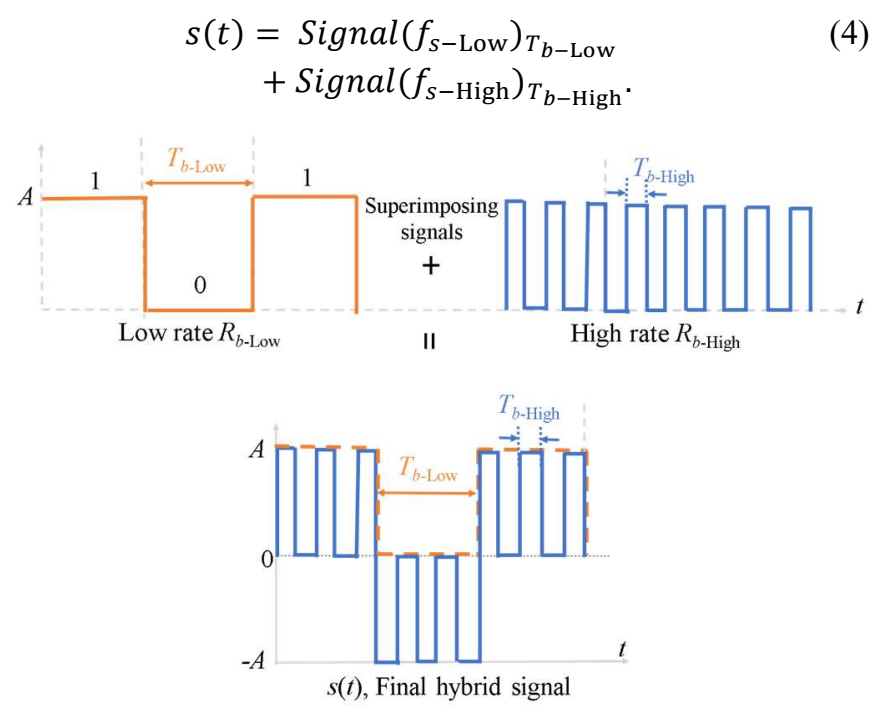

Fig. 2. Proposed modulation format for simultaneous transmission of high- and low-speed VLC-PD and OCC links, respectively.

To increase signal levels (i.e., $A$-depth) and therefore improve the BER performance as well as maintain the high quality of low-speed link, we provide a solution based on $A$ overlap $A_{\mathrm{OL}}$, see Fig. 3. As it will be shown in the experimental demonstration, this approach mitigates the frequency dependency of the high-pass filter (HPF) effect due to the use 
of bias-T in intensity modulation the LED with $s(t)$. Figure 3(a) shows $s(t)$ for $A$ oL of 0 , i.e., no overlap between $+A$ and $-A$ levels. Figures $3(\mathrm{~b})$ and (c) depict $s(t)$ with the overlapping regions. Note, higher $A_{\mathrm{OL}}$ leads to improved link performance for the high-speed VLC link at the cost of deteriorating performance deterioration the low-speed VLC (i.e., OCC) link. Thus, the need for optimization of signal overlapping. Note, more details on this will be given in the experimental part. The values for $A_{\text {High }}$ and $A_{\text {Low }}$ for high- and low-speed VLC links are given as:

$$
A_{\mathrm{High}}=A+\frac{A_{\mathrm{OL}}}{2}, A_{\mathrm{Low}}=A-\frac{A_{\mathrm{OL}}}{2},
$$

where $A_{\mathrm{OL}} / 2$ is due to the increase of $A_{\mathrm{OL}}$ for both positive and negative signal levels.

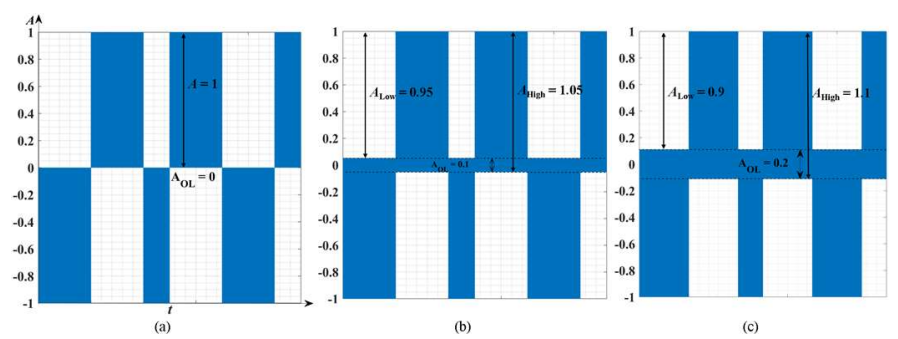

Fig. 3. Solution in terms of $A_{\mathrm{OL}}$ to reduce the effect of attenuation from HPF of bias-T on data recovery for $A_{\mathrm{OL}}$ of : (a) 0 , (b) 0.1 , and (c) 0.2 .

\section{B. SIMO hybrid VLC scheme: Experiment setup}

Figure 4 illustrates the block diagram of the proposed SIMO hybrid VLC scheme intended for used in OIoT. On the Tx side, a high-speed NRZ-OOK data stream with $5<R_{b \text {-High }}<70 \mathrm{Mb} / \mathrm{s}$ and a step of size $5 \mathrm{Mb} / \mathrm{s}$ is superimposed onto a 10-bit long low-speed data (i.e., [0011011001]) with $1<R_{b \text {-Low }}<10 \mathrm{~kb} / \mathrm{s}$ to produce $s(t) . s(t)$ generated in MATLAB, is uploaded to the arbitrary waveform generator (AWG-Teledyne test tools T3AWG3252 with a sampling frequency of $1 \mathrm{GS} / \mathrm{s}$ ) the output of which is used for intensity modulation of the LED via the bias-T (BT-A11). We have used a commercially available red LED with a peak wavelength $\lambda_{r}$ of $630 \mathrm{~nm}$ (Vishay VLMS1500GS08) with its power-current-voltage characteristics described in [6]. The LED was biased at a bias current $I_{b}$ of $90 \mathrm{~mA}$ with the transmit output power $P_{t}$ of $\sim 3.3 \mathrm{~mW}$ [6]. Table I shows the key parameters adopted in this work. The intensity modulated light signal is transmitted over a $1 \mathrm{~m}$ long free space channel via an optical lens, see the experimental setup in Fig. 4.

TABLE I

KEY EXPERIMENTAL PARAMETERS

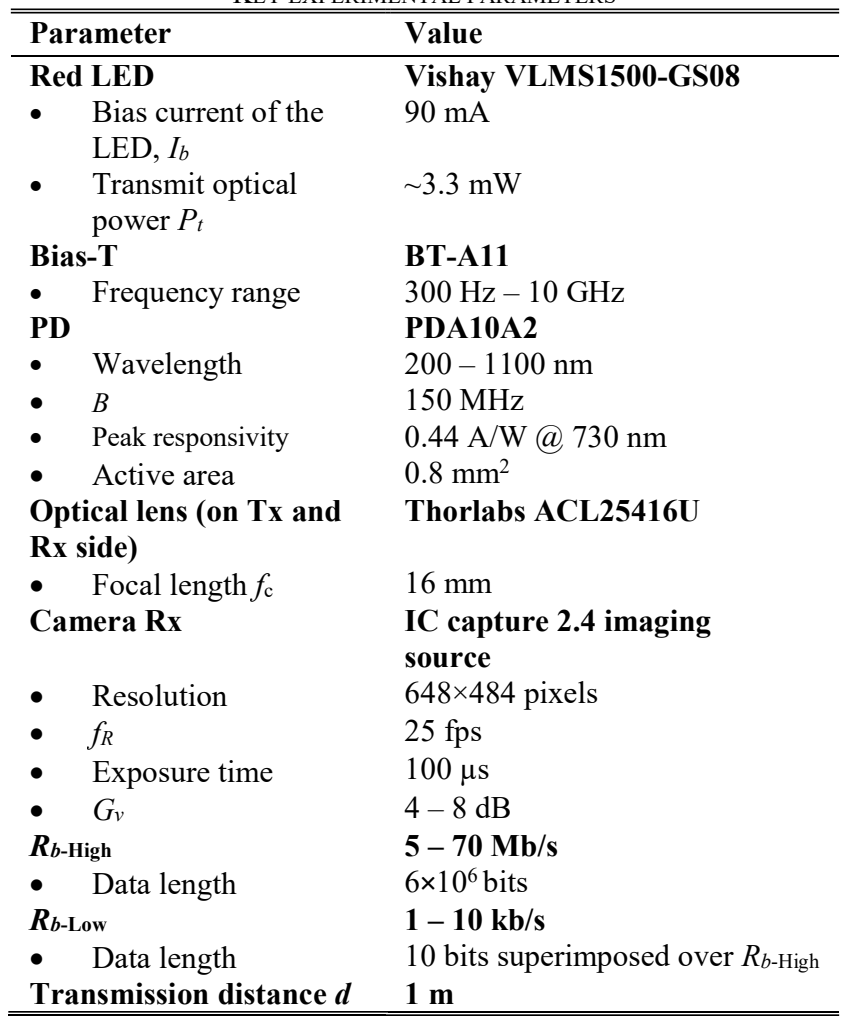

On the Rx side, we have used two types of detections: $(i)$ a CMOS RS-based IC capture camera with a pixel size $2.2 \times 2.2$ $\mu \mathrm{m}^{2}$; and (ii) a PD-based Rx (Thorlabs PDA10A2) connected to a real-time oscilloscope (Keysight MSDS104A with 20 $\mathrm{GSa} / \mathrm{s}$ sampling rate) for detecting the low- and high-speed signals, respectively. Following optical to electrical conversions, the regenerated signal at the output of IS-based Rx (OCC link) is given by:

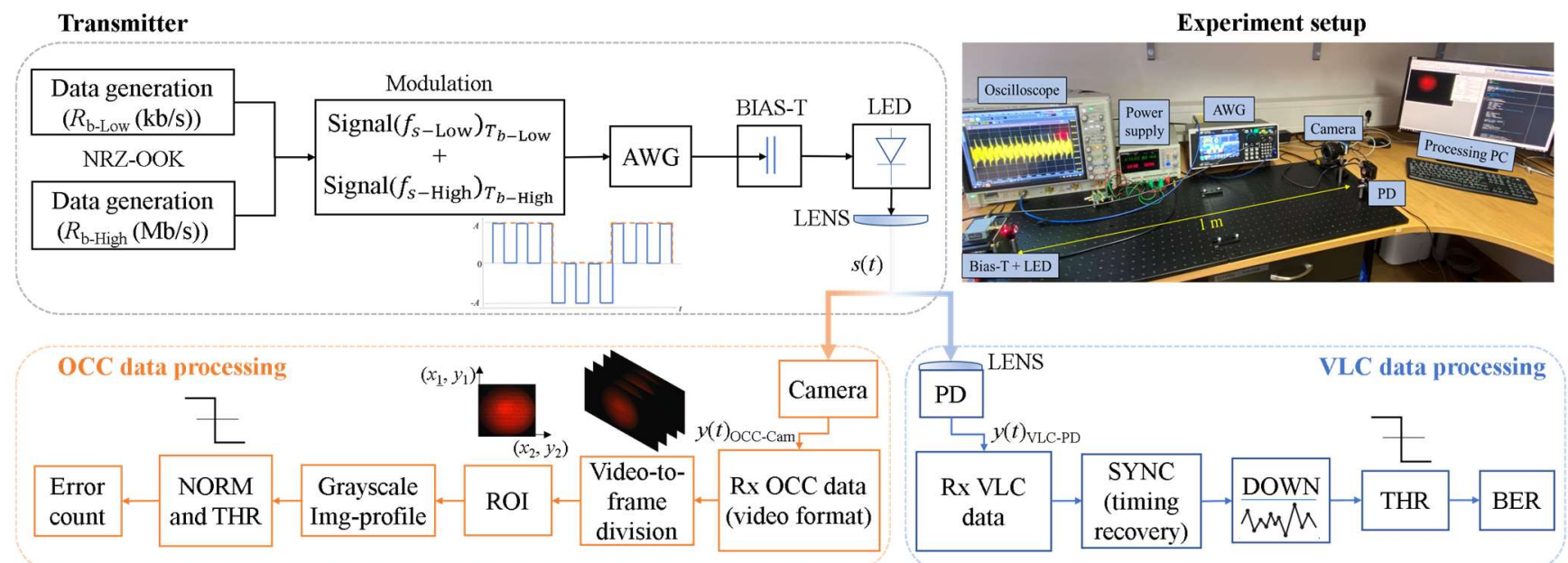

Fig. 4. Block diagram of the proposed OIoT: SIMO Hybrid VLC scheme including experiment setup inset. Note that, 'NORM', 'ROI', 'DOWN', 'THR' and 'BER' blocks refer to normalization, region-of-interest, down-sampling, thresholding and bit error rate, respectively. 


$$
y(t)_{\text {occ-Cam }}=\eta G_{v} s(t) \otimes h_{\mathrm{OCC}}(t)+n_{\mathrm{OCC}}(t),
$$

where $h_{\mathrm{OCC}}(t)$ is the combined impulse response of the channel and the camera, $\eta$ is the quantum efficiency of the camera, $\otimes$ is the time domain convolution, $n_{\mathrm{OCC}}(t)$ is the additive white Gaussian noise, which includes the ambient light induced shot noise and the noise in the camera such as fixed pattern, photocurrent shot, and flicker noise sources [24, 33], and $G_{v}$ presents the software defined global gain of the IS and the column amplifier block, which is given as [33, 34]:

$$
G_{v}(\mathrm{~dB})=20 \log _{10} \frac{V_{\mathrm{ADC}}}{V_{\text {pixels }}},
$$

where $V_{\mathrm{ADC}}$ is the voltage value, which is sampled by the analog-to-digital converter (ADC), and $V_{\text {pixles }}$ is the voltage obtained from the pixel integration of light during the exposure time. In the RS-based capturing mode, $(i)$ the camera sequentially integrates all illuminated pixels at the exposure time $t_{\text {row-exp }}$, which is the same as scanning [34,35]; and (ii) the readout time $t_{\text {read-out }}$ ensures that there is no overlapping of the rows of pixels, thus allowing multiple exposures of a single captured image. Note, $t_{\text {read-out }}$ protects rows of pixels from overlapping. In a single captured image, the RS scheme allows multiple exposures, which enables multiple incoming light states to be captured simultaneously within a single frame as each row is exposed once to the light. Using the RS effect of a CMOS camera is advantageous in ensuring flicker-free data transmission with increased $R_{b \text {-Low }}[35,36]$. In RS-based camera, the bit duration is given as:

$$
T_{b-\text { Low }}=t_{\text {row-exp }} \times N_{\text {row }},
$$

where $N_{\text {row }}$ is the pixel rows, which is based on the camera resolution.

The IS-based $\mathrm{Rx}$ was set to capture a video stream at $f_{R}$, resolution, interframe time and exposure time of $25 \mathrm{fps}$, $648 \times 484$ (red-green-blue (RGB)32) pixels, $\sim 5 \mathrm{~ms}$ and $100 \mu$ s, respectively. Note, a low-speed signal is captured by changing $G_{v}$ with the range of $4-8 \mathrm{~dB}$. The output of the camera is captured using the IC capture 2.4 software for further offline data processing in MATLAB based on traditional image processing techniques, see Fig. 4. As shown in Fig. 4, the captured signal in the form of a video stream is divided into image frames for further frame-by-frame processing to decode the received data. Next, in camera-based VLC the transmission distance $d$ between the LED and the camera needs considering, since as this distance increases, the size of region-of-interest (ROI) in the picture reduces, thus reducing the number of received messages per frame. ROI is defined as [37]:

$$
\mathrm{ROI}=\min \left(1, \frac{l_{\mathrm{LED}} \cdot f_{c}}{d \cdot l_{\mathrm{IS}}}\right)
$$

where $l_{\text {LED }}$ and $l_{\text {IS }}$ are the sizes of the LED and IS, respectively. By performing the ROI the coordinates, which define boundaries of the ROI in a full captured frame, are obtained as given by:

$$
\mathrm{ROI}=\operatorname{Img}\left\{\left(x_{1}, y_{1}\right) ;\left(x_{2}, y_{2}\right)\right\},
$$

where $\left(x_{1}, y_{1}\right)$ and $\left(x_{2}, y_{2}\right)$ are the top-left and bottom-right coordinates, respectively of the captured LED in the image frames, see Fig. 4. The ROI cropped image is then converted from RGB to the grayscale to retrieve the intensity profiles, which are normalized for thresholding and binarization of data frames and converting them to a vector transformation for decoding the data bit streams. Finally, the received data bit vector is compared with the transmitted data stream to ascertain the success of reception of received bits by determining the ratio of the wrongly decoded bits to the total number of transmitted bits (i.e., the number of bit errors). Note, the link performance can be improved by adopting the repeat packet strategy, where each packet is transmitted multiple times.

As for the high-speed VLC link, the regenerated signal at the output of PD is given as:

$$
y(t)_{\mathrm{VLC}-\mathrm{PD}}=\Re G_{\mathrm{PD}} s(t) \otimes h_{\mathrm{VLC}}(t)+n_{\mathrm{VLC}}(t),
$$

where $\Re$ is PD's responsivity, $G_{\mathrm{PD}}$ is PD's gain, $h_{\mathrm{VLC}}(t)$ is the combined transfer function of the channel and PD, and $n_{\mathrm{VLC}}(t)$ is the additive white Gaussian noise dominated by the background induced lights. The output of the optical Rx is applied to the synchronization module to determine the time delay between transmitted and received signals, followed by down-sampling and thresholding to recover the data, see Fig. 4. The received data bit stream is compared with the transmitted bits to determine the BER performance.

\section{EXPERIMENT RESULTS AND DISCUSSION}

As the main aim of the proposed scheme is to provide simultaneous data transmission at low- and high-speed, therefore it is necessary to study the inter-link impact on the system performance. This will be done in two stages.

1) The analysis of the attenuation effect on the PD-based VLC link due to the bias-T at $R_{b \text {-Low. }}$ A solution based on adjusting the $A$ level of the hybrid signal and increasing $R_{b}$ Low is proposed to improve the BER performance.

2) The effect of $R_{b \text {-High }}$ on low-speed signal is analyzed with respect to $A_{\mathrm{OL}}, R_{b \text {-Low }}$, and $G_{v}$.

\section{$A$. The effect of $R_{b \text {-Low }}$ on the VLC-PD Link}

We measured the frequency response of the bias-T using a spectrum analyzer (Rohde \& Schwarz FSW) to study its frequency dependent attenuation due to $R_{b \text {-Low }}$ on the highspeed link. The bias-T acts as an HPF, which attenuates the low frequency range, see Fig. 5. The insets in Fig. 5 illustrate the highly attenuated received high-speed signals for $1<R_{b \text {-Low }}<$ $10 \mathrm{~kb} / \mathrm{s}$. Thus, leading to the reduced $A_{\text {High }}$ depth and hence increased BER. Note, at higher frequencies the effect of bias-T is negligible, see the $1 \mathrm{MHz}$ signal in insets in Fig. 5. Therefore, to improve the BER performance, a solution based on $A_{\mathrm{OL}}>0$ was proposed in Section III, see Figs. 3(b) and (c). 


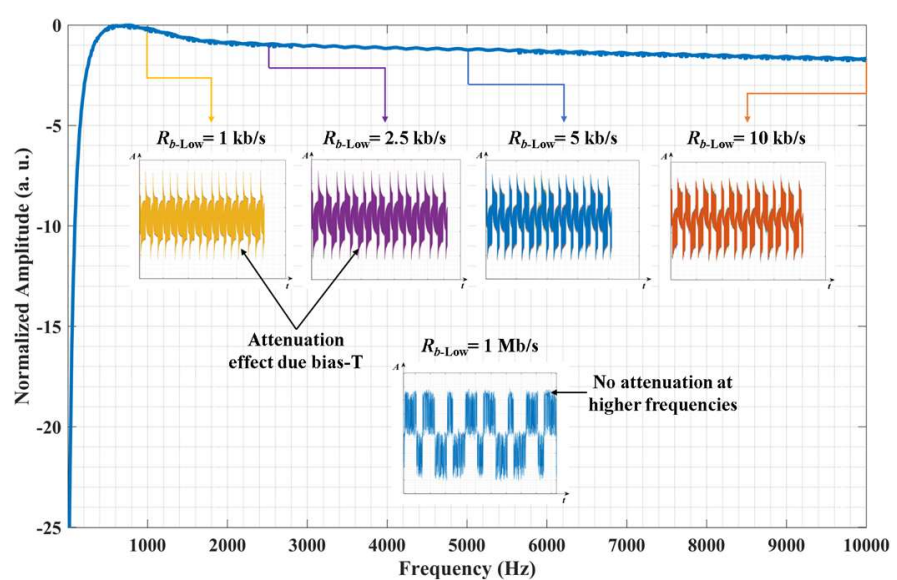

Fig. 5. The frequency response of bias-T and its attenuation effect on the received high-speed signal due to the low-speed signal. Insets show the received high-speed signal with and without attenuation effects with respect to varying $R_{b \text {-Low. }}$

For reference, we first measured the frequency response of the LED using spectrum analyzer (Rohde \& SchwarzFSW) with a $3-\mathrm{dB}$ bandwidth of $<50 \mathrm{MHz}$, see Fig. 6 [6], which is used as a Tx in the VLC link. For the high-speed link with the reference signal, we adopted a well-known strategy of zeropadded guard intervals as headers $[6,38]$ to obtain the realvalued signal. In this case, we achieved error-free transmission for $5<R_{b \text {-High }}<70 \mathrm{Mb} / \mathrm{s}$ with a step of size $5 \mathrm{Mb} / \mathrm{s}$ by transmitting $6 \times 10^{6}$ bits. Examples of captured eye diagrams at the $\mathrm{Rx}$ are illustrated in Fig. 7, where the two-level OOK signal show a wide eye-opening, i.e., error-free transmission. Note, the eye-opening reduces with increasing $R_{b \text {-High, which }}$ corresponds to the LEDs' frequency response (3 $\mathrm{dB} B_{\mathrm{LED}}<50$ $\mathrm{MHz}$ ) as shown in Fig. 6 [6].

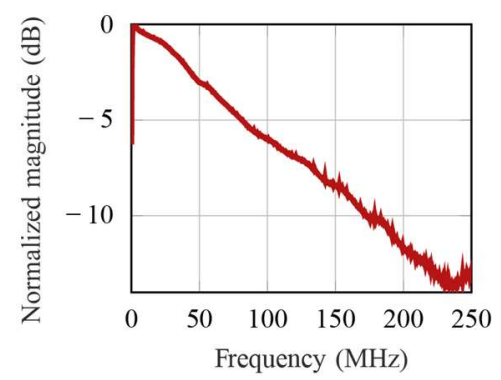

Fig. 6. Measured frequency response of the LED showing the 3-dB bandwidth slightly below $50 \mathrm{MHz}$.
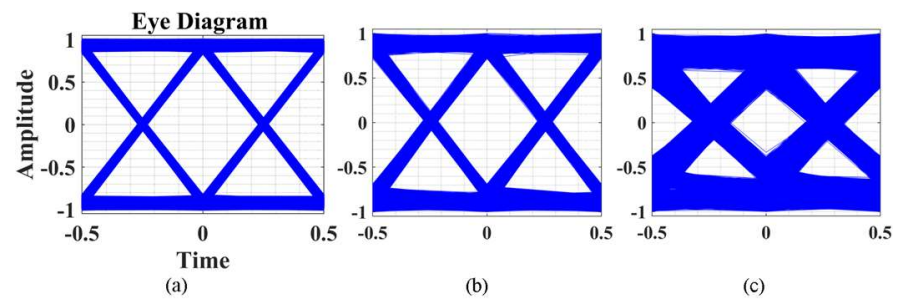

Fig. 7. The eye diagram of the received error free independent high-speed VLC link at $R_{b \text {-High }}$ for : (a) $5 \mathrm{Mb} / \mathrm{s}$, (b) $30 \mathrm{Mb} / \mathrm{s}$, and (c) $70 \mathrm{Mb} / \mathrm{s}$.

For high-speed link, every transmitted VLC data packets were saved and compared with the regenerated data patterns by separating the high and low bit of the low-speed signal with respect to 10-bit data sequence [0011011001]. To analyze the performance of high-speed PD-based VLC link with respect to low-speed OCC link in the proposed hybrid scheme, we have considered three main parameters: $(i) 5<R_{b \text {-High }}<70 \mathrm{Mb} / \mathrm{s}$ with a step of size $5 \mathrm{Mb} / \mathrm{s}$; (ii) $1<R_{b \text {-Low }}<10(1,2.5,5,10) \mathrm{kb} / \mathrm{s}$; and (iii) $0<A_{\mathrm{OL}}<0.2$ with a step of size 0.1 . Figure 8 (a) depicts the BER as a function of $R_{b \text {-High }}$ for the VLC-PD link and received hybrid signal for range of $A_{\mathrm{OL}}$ and $R_{b \text {-Low. It can be seen from }}$ Fig. 8(b) that, the $A$ depth of the received signal increases with $A_{\mathrm{OL}}$, which leads to the improved performance of VLC-PD. This corresponds to Eq. (5). As shown in Fig. 8(a), for $R_{b \text {-High }}$ of up to $70 \mathrm{Mb} / \mathrm{s}$; the BER plots are below the forward error correction (FEC) limit of $3.8 \times 10^{-3}$ for $R_{b \text {-Low }}$ of $10 \mathrm{~kb} / \mathrm{s}$ and $0<$ $A_{\mathrm{OL}}<0.2$. At the FEC BER limit for $R_{b \text {-Low }}$ of $5 \mathrm{~kb} / \mathrm{s}, R_{b \text {-High }}$ values are reduced by 7,10 , and $12 \mathrm{Mbps}$ for $A_{\mathrm{OL}}$ of $0.2,0.1$ and 0 , respectively. We observe more reduction in $R_{b \text {-High }}$ by about 33 and $51 \mathrm{Mbps}$ for $R_{b \text {-Low }}$ of 2.5 and $1 \mathrm{~kb} / \mathrm{s}$, respectively.

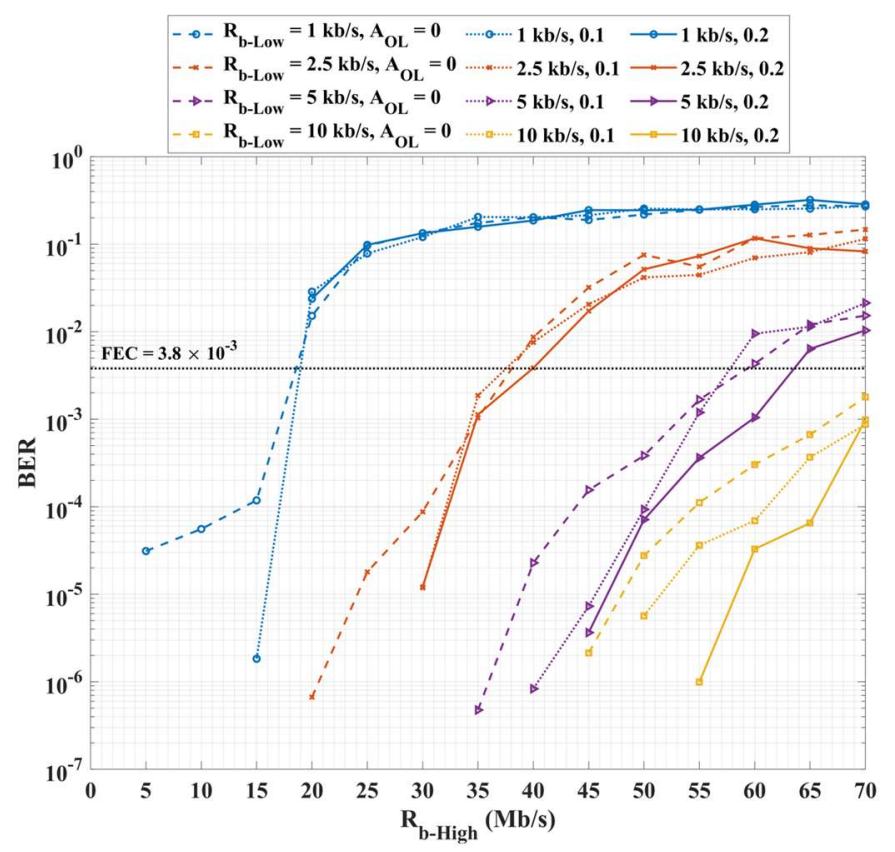

(a)
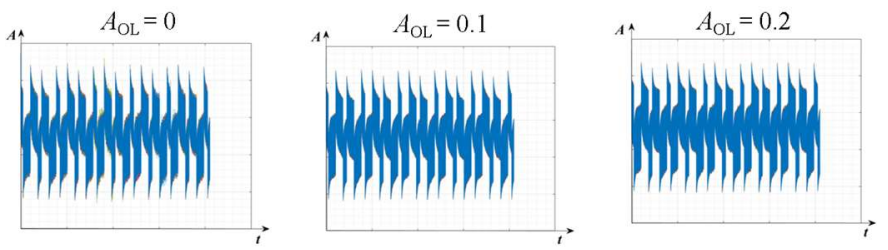

(b)

Fig. 8. Performance analysis: (a) BER versus the $R_{b \text {-High }}$ for VLC-PD link; and (b) received hybrid signal for a range of $R_{b \text {-Low }}$ and $A_{\mathrm{OL}}$.

To further improve the BER performance of VLC-PD, we carried out further measurement for $0.3<A_{\mathrm{OL}}<0.5$ with a step of size 0.1 , see Fig. 9(a). Note, according to Eq. (5), $A_{\text {Low }}$ decreases with the increasing $A_{\mathrm{OL}}$. On the other hand, further increase in $A_{\mathrm{OL}}$ significantly increases the $A$ depth of the received signal, see Fig. 9(b). It can be seen that, the FEC limits are met at around $R_{b \text {-High }}$ of $41 \mathrm{Mbps}$ for $R_{b \text {-Low }}$ of $2.5 \mathrm{~kb} / \mathrm{s}$ and $A_{\mathrm{OL}}$ of $0.3-0.5$. In Fig. 9(a), the curves at $R_{b \text {-Low }}$ of $2.5 \mathrm{kbps}$ and $A_{\mathrm{OL}}$ of 0.4 and 0.5 depict BER well below the forward error correction (FEC) limit until $R_{b \text {-High }}$ of $43 \mathrm{Mbps}$ and degrades

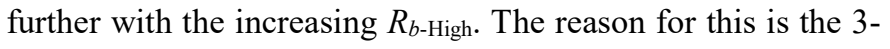


$\mathrm{dB}$ frequency response of the $\mathrm{LED}$, which is about $50 \mathrm{MHz}$, see Fig. 6 [6]. However, for lower values of $R_{b \text {-Low }}$ the BER plots are well above the FEC limits.

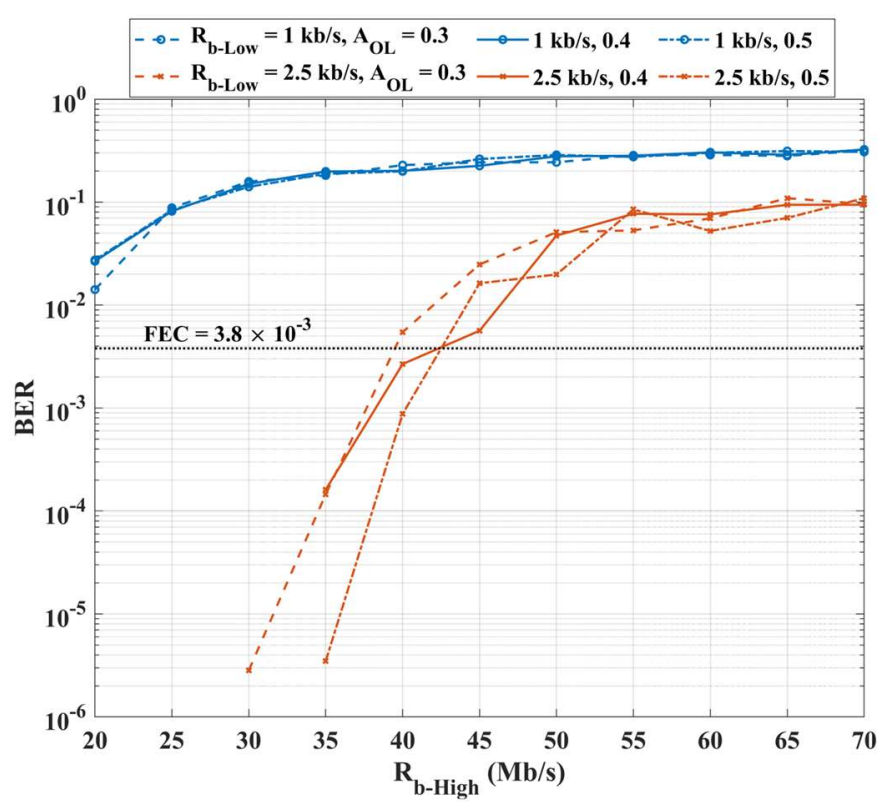

(a)
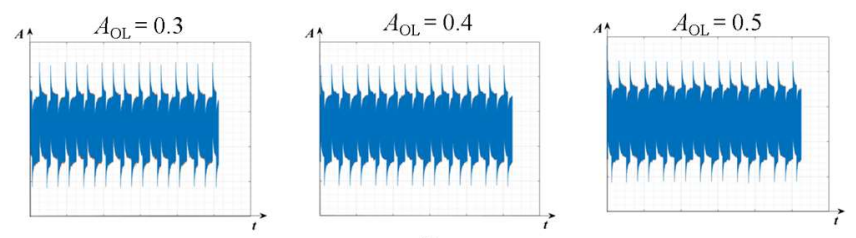

(b)

Fig. 9. Performance analysis: (a) BER performance as a function of $R_{b \text {-High }}$ for the VLC-PD link; and (b) received hybrid signal for $R_{b \text {-Low }}$ of 1 and $2.5 \mathrm{kbps}$ and $A_{\mathrm{OL}}$ of $0.3,0.4$, and 0.5 .

\section{B. The effect of $R_{b \text {-High }}$ on Low-speed OCC (IS-based VLC) link}

Increasing $A_{\mathrm{OL}}$ improves the performance of the PD-based VLC link at the cost of reduced $A_{\text {Low }}$ levels, see Eq. (5). This results in reduced intensity levels between 'high' and 'low' of the low-speed signal in the received image frame. Therefore, it is essential to analyze the performance of OCC link considering the high-speed VLC, $A_{\mathrm{OL}}$, and $G_{v}$.

Like VLC-PD, first, we considered only the OCC link for 1 $<R_{b \text {-Low }}<10$ (i.e., $1,2.5,5,10$ ) $\mathrm{kb} / \mathrm{s}$ and captured a $4 \mathrm{~s}$ video stream (i.e., $25 \mathrm{fps}$ ) of the intensity modulated LED. In every image frame, $4,10,20$, and 22 repeated data packets of 10-bit long [0011011001] were captured at $R_{b \text {-Low }}$ of $1,2.5,5$ and 10 $\mathrm{kb} / \mathrm{s}$, respectively. Considering that, a smaller number of bits were transmitted the OCC link performance is analyzed in terms of the reception success, which is defined as the ratio of incorrectly decoded bits to the total number of transmitted bits. We have achieved 100,97 , and $75 \%$ reception success rates for $R_{b \text {-Low }}$ of 1 and $2.5,5$, and $10 \mathrm{~kb} / \mathrm{s}$, respectively.

The performance of OCC link in the hybrid scheme is studied for $R_{b \text {-High }}, R_{b \text {-Low }}, A_{\mathrm{OL}}$, and $4<G_{v}<8 \mathrm{~dB}$, see Fig. 10 . It can be seen that, the intensity levels of the received low-speed signal are marginally affected for higher values of $A_{\mathrm{OL}}$, which corresponds to the reduced $A_{\text {Low }}$ as shown in Fig. 3 and Eq. (5), see Fig. 10(a). Figure 10(b) illustrates the normalized intensity profiles for $A_{\mathrm{OL}}$ of 0 and 0.2 as well as the threshold levels based on the mean of the high and low normalized intensities. Note, the threshold level can be adaptively set based on the intensity levels in the image frame.
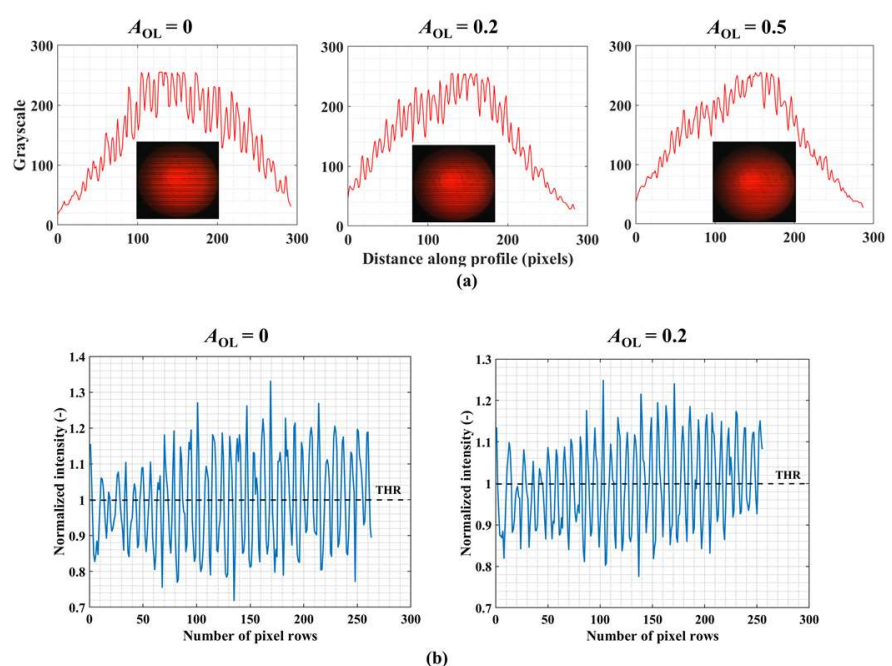

Fig. 10. Effect of $A_{\mathrm{OL}}$ on the low-speed OCC link at $R_{b \text {-Low }}=2.5 \mathrm{~kb} / \mathrm{s}$ and $G_{v}=$ $8 \mathrm{~dB}$ for: (a) grayscale, and (b) normalized intensity profiles.

Figure 11 demonstrates the gray scale levels of low-speed OCC with $R_{\mathrm{b}-\text { Low }}$ and $G_{v}$ of $5 \mathrm{~kb} / \mathrm{s}$ and $4 \mathrm{~dB}$, respectively for $R_{b \text {-High }}$ of 10 and $70 \mathrm{Mb} / \mathrm{s}$. It shows higher levels of amplitude fluctuation at $R_{b \text {-High }}$ of $10 \mathrm{Mb} / \mathrm{s}$ compared with $R_{b \text {-High }}$ of 70 $\mathrm{Mb} / \mathrm{s}$. This is because of the higher number of bits being superimposed per bit of $R_{b \text {-Low }}$ for $R_{b \text {-High }}$ of $70 \mathrm{Mb} / \mathrm{s}$ compared with $R_{b \text {-High }}$ of $10 \mathrm{Mb} / \mathrm{s}$, see blue waveforms in Figs. 11(a) and (b).
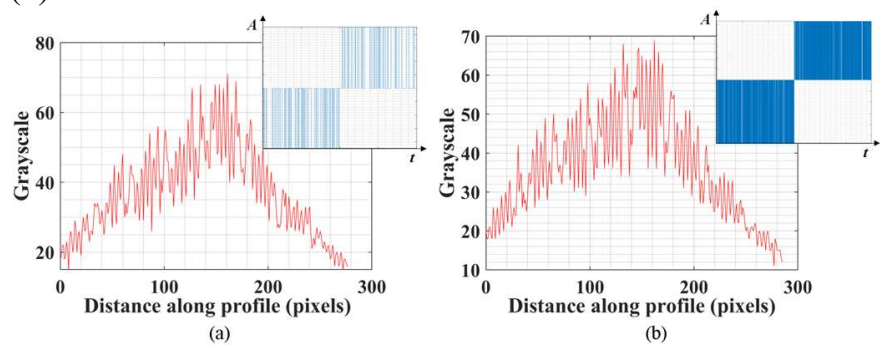

Fig. 11. Effect of high-speed VLC on OCC link at $R_{b \text {-Low }}=5 \mathrm{~kb} / \mathrm{s}$ and $G_{v}=$ $4 \mathrm{~dB}$ for $R_{b \text {-High }}$ of: (a) $10 \mathrm{Mb} / \mathrm{s}$, and (b) $70 \mathrm{Mb} / \mathrm{s}$. Note, blue waveform in insets represents the transmitted signal at respective $R_{b \text {-Low }}$ and $R_{b \text {-High }}$.

Next, we have investigated the performance of OCC link in terms of the reception success for $R_{\mathrm{b}-\mathrm{Low}}$ of 1 and $2.5 \mathrm{~kb} / \mathrm{s}$. The $100 \%$ (error-free transmission) reception success is achieved at $R_{\mathrm{b}-\mathrm{Low}}$ of $1 \mathrm{~kb} / \mathrm{s}$ for $0<A_{\mathrm{OL}}<0.2$ and $G_{v}$ of $4-8 \mathrm{~dB}$. Therefore, for further analysis as in the VLC-PD link, we considered higher values of $A_{\mathrm{OL}}$ (i.e., $\left.0.3<A_{\mathrm{OL}}<0.5\right)$ for $R_{\mathrm{b} \text {-Low }}$ of 1 and $2.5 \mathrm{~kb} / \mathrm{s}$, see Figs. 12(a) and (b) (note, error-free plots, i.e., $100 \%$ reception success plots are not shown in Fig. 12(a)). It can be seen that, at $G_{v}$ of $4 \mathrm{~dB}$ the reception success rates are above 95 and $94 \%$ for 1 and $2.5 \mathrm{~kb} / \mathrm{s}$, respectively. From Fig. 12 , the performance of OCC links degrades with increase in $G_{v}$ and $A_{\mathrm{OL}}$ (this corresponds to Figs. 9 and 3 and Eqs. (5) and (7)). In our recent works [33], we investigated how higher values of $G_{v}$ ensured reception of the low-speed signal, see Eq. (7), even 


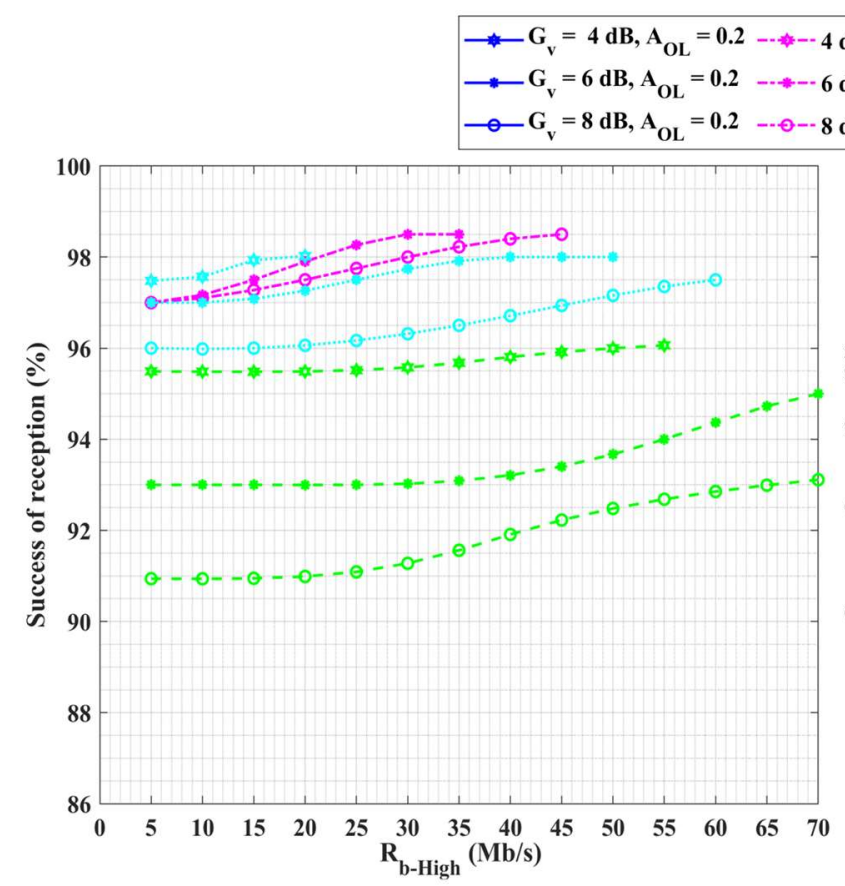

(a)
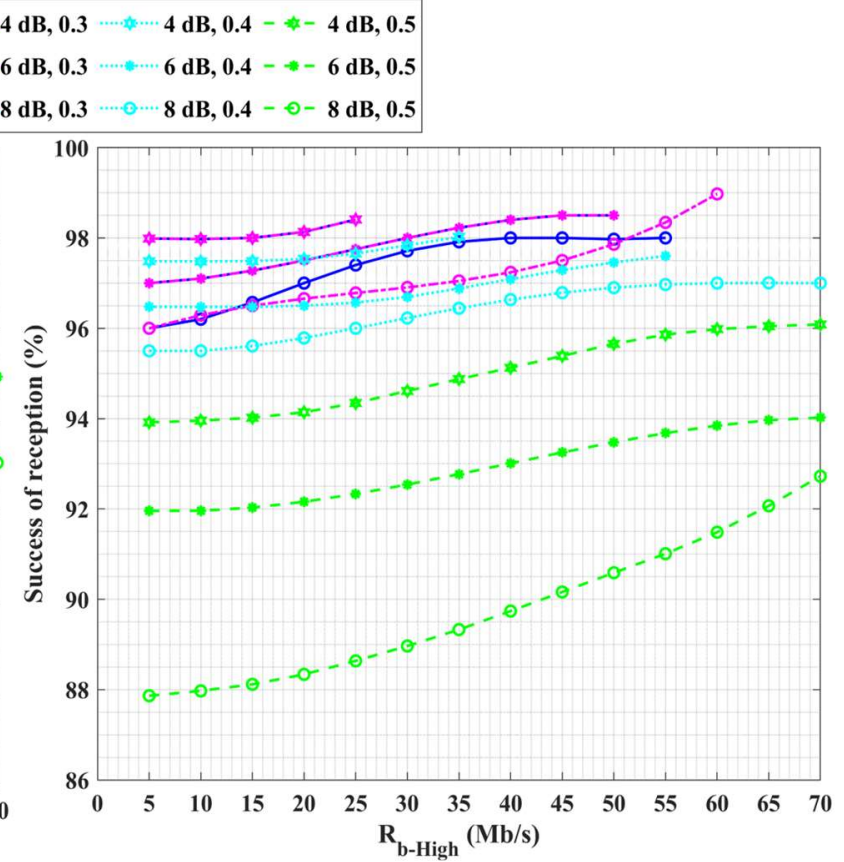

(b)

Fig. 12. Performance of OCC link in the proposed hybrid scheme: the success of reception performance as a function of $R_{b \text {-High }}, G_{v}$ and $A_{\mathrm{OL}}$ for $R_{b \text {-Low }}$ of: (a) $1 \mathrm{~kb} / \mathrm{s}$, and (b) $2.5 \mathrm{~kb} / \mathrm{s}$.

in outdoor envirnoments under turbulence and fog conditions. This is because increasing $G_{v}$ improves signal amplification passing through the camera ADC and to be focused on the IS, which results in intensity saturation within the captured image frames. Therefore, in this work we carry out analysis for the OCC link by changing $G_{v}$ that can be further beneficial in multiTx-based hybrid schemes. For the system with a line-of-sight propagation path with much reduced level of ambient light at the $\operatorname{Rx}$ (i.e., $\sim 4$ lux $\left( \pm 0.2\right.$ lux )), increasing $G_{v}$ results in saturation of the focused LED light in the captured image frame. Thus leading to reduced $A$ levels of the received 1 and 0 bits on the RS captured frame. However, it can be seen from Fig. 12 that, an reception success $>91$ and $88 \%$ are achieved even for higher values of $G_{v}$ of $8 \mathrm{~dB}$ and $A_{\mathrm{oL}}$ of 0.5 for $R_{b \text {-Low }}$ of 1 and $2.5 \mathrm{~kb} / \mathrm{s}$, respectively.

Figure 13 depicts the reception success versus $R_{b \text {-High }}$ of the OCC link for $R_{b \text {-Low }}$ of $5 \mathrm{~kb} / \mathrm{s}$ for range of $G_{v}$ and $A_{\mathrm{OL}}$. It can be seen that, the lowest and highest reception success rates are within the band of $88-92 \%$ and $92.5-94 \%$ within the $4<G_{v}<$ $8 \mathrm{~dB}$ and $0<A_{\mathrm{OL}}<0.2$, respectively for $R_{b \text {-High }}$ of $5-70 \mathrm{Mb} / \mathrm{s}$. Considering that $f_{R}$ of the IS-based $\mathrm{Rx}$ is limited, increasing $R_{b \text { - }}$ Low degrades the performance of OCC link. If $T_{b \text {-Low }}$ exceeds $t_{\text {read-out }}$ of the camera, it leads to more transition frames, increased overlap between the captured data rows and higher aliasing, thus, degrading the performance of the link. For example, at $R_{b \text {-Low }}$ of $10 \mathrm{~kb} / \mathrm{s}$, the measured reception success rate is dropped to $65-70 \%$ for $G_{v}$ of $4 \mathrm{~dB}, A_{\mathrm{OL}}$ of 0 , and for 5 $<R_{b-\mathrm{High}}<70 \mathrm{Mb} / \mathrm{s}$.

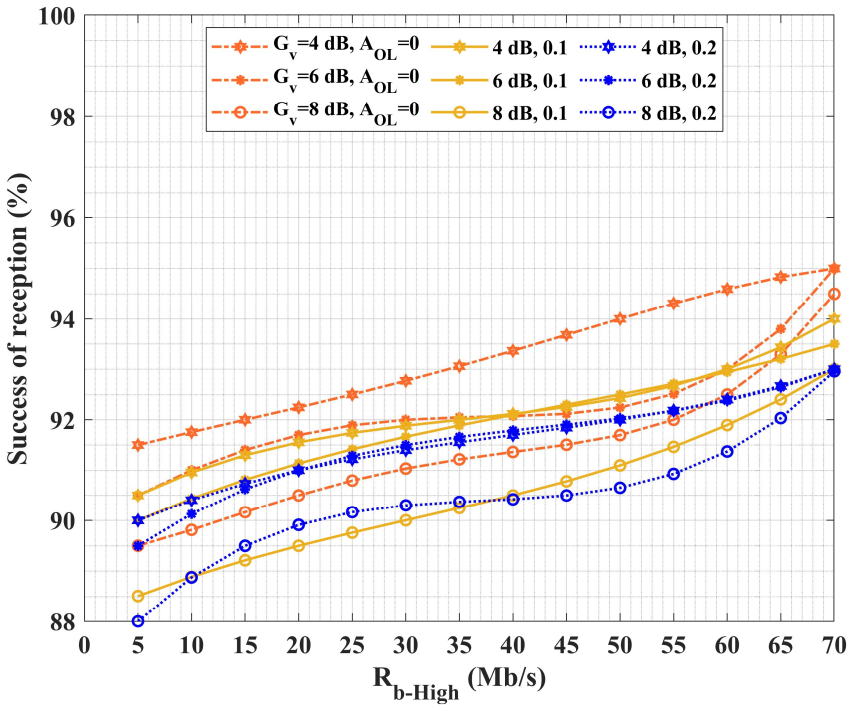

Fig. 13. The reception success rate for the OCC link in the proposed hybrid scheme: the success of reception at $R_{b-\text { Low }}=5 \mathrm{~kb} / \mathrm{s}$.

\section{SUMMARY OF RESULTS}

We summarized the performance of the proposed SIMO hybrid VLC scheme based on the results obtained as follow:

1) A solution to overcome the attenuation effect from the bias- $T$ used to drive the LED in terms of $A_{\mathrm{OL}}$ was provided. The effect of $A_{\mathrm{OL}}$ on both high- and low-speed PD- and ISbased VLC links, respectively was analyzed as:

(i) $A_{\text {OL }}$ helps to improve the BER performance of PDbased VLC link by increasing the $A$ depth of the hybrid signal. For example, at $0<A_{\mathrm{OL}}<0.2 \mathrm{BER}$ below the FEC limit of $3.8 \times 10^{-3}$ is measured for $R_{b \text {-Low }}$ of 2.5 , and $5 \mathrm{~kb} / \mathrm{s}$ at reduced $R_{b \text {-High }}$ of 10 and $35 \mathrm{Mb} / \mathrm{s}$, respectively. While, at $0.3<A_{\mathrm{OL}}<0.5$ the BER FEC 
limits are met at $R_{\mathrm{b}-\mathrm{High}}$ of $41 \mathrm{Mbps}$ for $R_{b \text {-Low }}$ of $2.5 \mathrm{~kb} / \mathrm{s}$.

(ii) For the IS-based VLC link (i.e., OCC), increasing $A_{\mathrm{OL}}$ reduces the total $A_{\text {Low }}$ level, thus reducing the difference between 'high' and 'low' intensity levels of the captured data in the image frame. However, to improve the OCC performance even for higher $A_{\mathrm{OL}}$, a solution is proposed to use the minimum $G_{v}$ to limit the incoming light and avoid the saturation effect on the received low-speed signal in the image frame. For example, at $G_{v}$ of $4 \mathrm{~dB}, 0<A_{\mathrm{OL}}<0.2$ and $5<R_{b \text {-High }}<$ $70 \mathrm{Mb} / \mathrm{s}$, the reception success of $>96$ and $90 \%$ are measured at $R_{b \text {-Low }}$ of 2.5 and $5 \mathrm{~kb} / \mathrm{s}$, respectively.

(iii) We also analyzed the performance of the OCC link at higher $G_{v}$ for implementation of the future multi$\mathrm{Tx} /$ light source-based hybrid scheme to overcome the noise effect. It was shown that, for $5<R_{b \text {-High }}<$ $70 \mathrm{Mb} / \mathrm{s}$ the reception success of $>88 \%$ is measured at $G_{v}$ of $8 \mathrm{~dB}$ for $R_{b \text {-Low }}$ of $2.5 \mathrm{~kb} / \mathrm{s}\left(0<A_{\mathrm{OL}}<0.5\right)$ and $5\left(0<A_{\mathrm{OL}}<0.2\right) \mathrm{kb} / \mathrm{s}$.

Therefore, it is necessary to balance the performance of both high- and low-speed PD- and IS-based VLC links in the proposed hybrid scheme by providing the optimized solution to improve individual performances regardless of the bias-T attenuation and $A_{\mathrm{OL}}$ effects on the VLC and OCC links, respectively.

2) From the summary of results presented based on the performance analysis, $0<A_{\mathrm{OL}}<0.2, R_{b \text {-Low }}$ of 2.5 and $5 \mathrm{~kb} / \mathrm{s}, 5<R_{b \text {-High }}<60 \mathrm{Mb} / \mathrm{s}$, and $G_{v}$ of $4 \mathrm{~dB}$ can be considered as the parameters with optimum values derived from the experimental demonstration of the proposed SIMO hybrid VLC scheme for OIoT environments. These values can help further implementation of the proposed scheme in real and extended OIoT environments.

3) Based on the summary of results, we have proposed a solution for the limitations within hybrid VLC-OCC schemes [28-31] described in Section II Related works.

(i) We have proposed a hybrid modulation scheme for transmission of high- and low-speed data simultaneously using a single LED source which was not investigated in $[28,29]$.

(ii) The bandwidth of the hybrid VLC/OCC scheme in [30] was limited thus the data rates of $1.67 \mathrm{kbps}\left(R_{b-}\right.$ Low $)$ and $100 \mathrm{kbps}\left(R_{b-\mathrm{High}}\right)$ were reported for the OCC and VLC links, respectively. This issue was addressed using a red chip LED Tx, with the option of adding green and blue LEDs to produce a white light for illumination and data communications using a red chip LED and the proposed hybrid modulation format. Based on this, we have achieved $R_{b \text {-Low }}$ of 2.5 and $5 \mathrm{~kb} / \mathrm{s}$ and $5<R_{b-\mathrm{High}}<60 \mathrm{Mb} / \mathrm{s}$.

(iii) As in [31], changing the power levels corresponding to the varying amplitude depths of the data bit stream affects the signal detection process, which is not investigated. In the proposed SIMO hybrid VLC scheme, we have provided detailed data processing for high- and low-speed signals captured using PD- and IS-based Rxs along with $A_{\text {OL }}$ solution to improve the
BER performance of PD-based VLC link by increasing the $A$ depth of the hybrid signal, and using the optimal $G_{v}$ of $4 \mathrm{~dB}$ to improve the performance of IS-based VLC link.

\section{CONCLUSIONS AND FUTURE OUTLOOK}

This paper demonstrated experimental implementation of OIoT: the SIMO hybrid VLC scheme in indoor static environment. A single red LED-based Tx with a bias-T was used for simultaneous transmission of high- and low-speed signals. For this reason, we proposed a hybrid modulation scheme with $R_{b \text {-High }}$ and $R_{b \text {-Low }}$ of up to $60 \mathrm{Mb} / \mathrm{s}$ and $5 \mathrm{~kb} / \mathrm{s}$ for high-speed VLC and low-speed OCC links, respectively. The results demonstrated that, increasing $R_{b \text {-High }}$ and $R_{b \text {-Low }}$ improved the OCC and VLC performance, respectively. As part of the modulation format, we introduced the concept of the $A_{\mathrm{OL}}$ to increase the transmission rate, and reduce the bias- $T$ induced attenuation. We addressed the problems of bandwidth efficiency, the effect of multilevel LED transmission due to $A_{\mathrm{OL}}$ and provided a comprehensive analysis of data processing for the PD- and IS-based VLC links. It is envisioned that; the proposed SIMO hybrid VLC scheme can provide versatile OIoT-based indoor services that can allow users to receive data regardless of their devices.

Within the hybrid transmission environment, high-speed VLC links can be used for large amount of data downloading, internet surfing, and online streaming, while the low-speed links can be used to transmit short messages, identification and promotion information, control signals, and indoor localization within OIoT environments. As a part of potential future development of the proposed scheme in practical scenarios, green and blue colors can be added to the red LED to produce a white light for illumination while still offering data communications via the red chip LED. In this way, the proposed scheme can overcome the problem of bandwidth efficiency. Equalization techniques [39, 40] and different modulation formats such as OFDM [41] can be implemented to improve the reception success rate and the BER performance for low-speed and high-speed VLC links, respectively. The scheme can also be tested for various transmission ranges between the LED and camera- and PD-based Rxs along with different angular orientations to support mobility in OIoT environments.

\section{REFERENCES}

[1] H. Aksu, L. Babun, M. Conti, G. Tolomei and A. S. Uluagac, "Advertising in the IoT Era: Vision and Challenges," IEEE Communications Magazine, vol. 56, no. 11, pp. 138-144, 2018.

[2] N. Wang, P. Wang, A. Alipour-Fanid, L. Jiao and K. Zeng, "PhysicalLayer Security of $5 \mathrm{G}$ Wireless Networks for IoT: Challenges and Opportunities," IEEE Internet of Things Journal, vol. 6, no. 5, pp. 81698181 , Oct. 2019.

[3] IEEE Standard for Local and Metropolitan Area Networks_Part 15.7: Short-Range Wireless Optical Communication Using Visible Ligh, IEEE Standard 802.15.7, Dec. 2018. [Online]. Available: http://standards.ieee.org/_ndstds/standard/802.15.7-2011.html

[4] Z. Ghassemlooy, S. Zvanovec, Moh. A. Khalighi, W. O. Popoola, J. Perez, "Optical wireless communication systems", Optik, vol. 151, pp. 16, 2017.

[5] P. H. Pathak, X. Feng, P. Hu, and P. Mohapatra, "Visible light communication, networking, and sensing: A survey, potential and challenges," 
IEEE Commun. Surveys Tuts., vol. 17, no. 4, pp. 2047-2077, 4th Quart., 2015.Z. Ghassemlooy, L.N. Alves, S. Zvanovec, and M.A. Khalighi, eds. Visible Light Communications: Theory and Applications, CRC Press, 2017.

[6] P. Chvojka, et al., "Visible light communications: increasing data rates with polarization division multiplexing," Opt. Lett. Vol. 45, pp. 2977$2980,2020$.

[7] I.-C. Lu, C.-H. Yeh, D.-Z. Hsu, and C.-W. Chow, "Utilization of 1-GHz VCSEL for 11.1-Gbps OFDM VLC wireless communication," IEEE Photonics Journal, vol. 8, no. 3, Jun. 2016.

[8] M. S. Islim et al., "Towards $10 \mathrm{~Gb} / \mathrm{s}$ orthogonal frequency division multiplexing-based visible light communication using a $\mathrm{GaN}$ violet micro-LED," Photon. Res., vol. 5, no. 2, pp. A35-A43, 2017.

[9] R. Bian, I. Tavakkolnia and H. Haas, "15.73 Gb/s Visible Light Communication With Off-the-Shelf LEDs," Journal of Lightwave Technology, vol. 37, no. 10, pp. 2418-2424, 15 May15, 2019.

[10] S. Rajagopal, R. D. Roberts, and S. K. Lim, "IEEE 802.15.7 visible light communication: modulation schemes and dimming support," IEEE Communications Magazine, vol. 50, no. 3, pp. 72-82, 2012.

[11] I. Demirkol, D. Camps-Mur, J. Paradells, M. Combalia, W. Popoola and H. Haas, "Powering the Internet of Things through Light Communication," IEEE Communications Magazine, vol. 57, no. 6, pp. 107-113, 2019.

[12] C. Chen, S. Fu, X. Jian, M. Liu, X. Deng and Z. Ding, "NOMA for Energy-Efficient LiFi-Enabled Bidirectional IoT Communication," IEEE Transactions on Communications, vol. 69, no. 3, pp. 1693-1706, 2021.

[13] Y. Chen, S. Li and H. Liu, "Dynamic Frequency Reuse Based on Improved Tabu Search in Multi-User Visible Light Communication Networks," IEEE Access, vol. 7, pp. 35173-35183, 2019.

[14] Y. Hou, S. Xiao, H. Zheng and W. Hu, "Multiple access scheme based on block encoding time division multiplexing in an indoor positioning system using visible light," IEEE/OSA Journal of Optical Communications and Networking, vol. 7, no. 5, pp. 489-495, May 2015.

[15] J. Sanusi, S. Idris, A. M. Aibinu, S. Adeshina and A. N. Obadiah, "Handover in Hybrid LiFi and WiFi Networks," 2019 15th International Conference on Electronics, Computer and Computation (ICECCO), Abuja, Nigeria, 2019, pp. 1-6.

[16] S. R. Teli, S. Zvanovec and Z. Ghassemlooy, "Optical Internet of Things within 5G: Applications and Challenges," 2018 IEEE International Conference on Internet of Things and Intelligence System (IOTAIS), Bali, pp. 40-45, 2018.

[17] J. Y. Sung, C. W. Chow, C. H. Yeh, and Y. C. Wang, "Service integrated access network using highly spectral-efficient MASK-MQAM-OFDM coding," Opt. Express, vol. 21, pp. 6555-6560, 2013.

[18] C. Chen, W. D. Zhong, D. Wu, "Integration of variable-rate OWC with OFDM-PON for hybrid optical access based on adaptive envelope modulation," Optics Communications, vol. 381, pp. 10-17, 2016.

[19] M. J. Jang, "IEEE 802.15 WPAN 15.7 amendment-optical camera communications study group (SG 7a)," (2021 [Online accessed 9 February 2021]).

[20] S. Teli, W. A. Cahyadi and Y. H. Chung, "Optical Camera Communication: Motion over Camera," IEEE Communications Magazine, vol. 55, no. 8, pp. 156-162, Aug. 2017.

[21] T. Le, N. Le and Y. M. Jang, "Performance of rolling shutter and global shutter camera in optical camera communications," 2015 International Conference on Information and Communication Technology Convergence (ICTC), Jeju, 2015, pp. 124-128.

[22] X. Liu, X. Wei and L. Guo, "DIMLOC: Enabling High-Precision Visible Light Localization Under Dimmable LEDs in Smart Buildings," IEEE Internet of Things Journal, vol. 6, no. 2, pp. 3912-3924, April 2019.

[23] S. Ma, Q. Liu and P. C. -. Sheu, "Foglight: Visible Light-Enabled Indoor Localization System for Low-Power IoT Devices," IEEE Internet of Things Journal, vol. 5, no. 1, pp. 175-185, Feb. 2018.

[24] S. R. Teli, S. Zvanovec, R. Perez-Jimenez and Z. Ghassemlooy, "Spatial frequency-based angular behavior of a short-range flicker-free MIMOOCC link," Applied Optics, vol. 59, no. 33, pp. 10357-10368, 2020.

[25] R. Boubezari, H. Le Minh, Z. Ghassemlooy and A. Bouridane, "Smartphone Camera Based Visible Light Communication,"Journal of Lightwave Technology, vol. 34, no. 17, pp. 4121-4127, 1 Sept.1, 2016.

[26] S. Mangold, "Research team demonstrates LEDs that use visible light to talk to each other and internet," [Online] Available: https://phys.org/news/2015-09-team-visible-internet.html\#jCp

[27] H. Yang, W. Zhong, C. Chen, A. Alphones and P. Du, "QoS-Driven Optimized Design-Based Integrated Visible Light Communication and
Positioning for Indoor IoT Networks," IEEE Internet of Things Journal, vol. 7, no. 1, pp. 269-283, Jan. 2020.

[28] M. K. Hasan, M. Shahjalal, M. Z. Chowdhury, M. T. Hossan, and Y. M. Jang, "Fuzzy logic based network selection in hybrid OCC/Li-Fi communication system," in Proc. 10th Int. Conf. Ubiquitous Future Netw. (ICUFN), Prague, Czech Republic, Jul. 2018, pp. 95-99.

[29] M. K. Hasan, M. Z. Chowdhury, M. Shahjalal, and Y. M. Jang, "Fuzzy based network assignment and link-switching analysis in hybrid OCC/LiFi system," Wireless Commun. Mobile Comput., vol. 2018, Nov. 2018, Art. no. 2870518.

[30] D. T. Nguyen, S. Park, Y. Chae and Y. Park, "VLC/OCC Hybrid Optical Wireless Systems for Versatile Indoor Applications," IEEE Access, vol. 7, pp. 22371-22376, 2019.

[31] M. K. Hasan, N. T. Le, M. Shahjalal, M. Z. Chowdhury and Y. M. Jang, "Simultaneous Data Transmission Using Multilevel LED in Hybrid OCC/LiFi System: Concept and Demonstration," IEEE Communications Letters, vol. 23, no. 12, pp. 2296-2300, Dec. 2019.

[32] S. R. Teli, S. Zvanovec and Z. Ghassemlooy, "The first tests of smartphone camera exposure effect on optical camera communication links," 2019 15th International Conference on Telecommunications (ConTEL), Graz, Austria, pp. 1-6, 2019.

[33] V. Matus, E. Eso, S. R. Teli, R. Perez-Jimenez and S. Zvanovec, "Experimentally derived feasibility of optical camera communications under turbulence and fog conditions," SENSORS, vol. 20, pp.1-15, 2020.

[34] T. Kuroda, "Essential principles of image sensors," CRC press, 2017.

[35] C.-W. Chow, C.-Y. Chen, and S.-H. Chen, "Visible light communication using mobile-phone camera with data rate higher than frame rate," Opt. Express, vol. 23, pp. 26080-26085, 2015.

[36] V.P. Rachim and W. Chung, "Multilevel intensity-modulation for rolling shutter-based optical camera communication," IEEE Photonics Technology Letters, vol. 30, pp. 903-906, 2018.

[37] A. Duque, R. Stanica, H. Rivano, A. Desportes, "Analytical and simulation tools for optical camera communications," Computer Communications, vol. 160, pp. 52-62, 2020.

[38] W. Ozan, R. Grammenos and I. Darwazeh, "Zero Padding or Cyclic Prefix: Evaluation for Non-Orthogonal Signals," IEEE Communications Letters, vol. 24, no. 3, pp. 690-694, 2020.

[39] O. I. Younus et al., "Data Rate Enhancement in Optical Camera Communications Using an Artificial Neural Network Equaliser," IEEE Access, vol. 8, pp. 42656-42665, 2020.

[40] X. Li, Z. Ghassemlooy, S. Zvanovec, R. Perez-Jimenez and P. A. Haigh, "Should Analogue Pre-Equalisers be Avoided in VLC Systems?" IEEE Photonics Journal, vol. 12, no. 2, pp. 1-14, April 2020.

[41] T. Nguyen, M. D. Thieu and Y. M. Jang, "2D-OFDM for Optical Camera Communication: Principle and Implementation," IEEE Access, vol. 7, pp. 29405-29424, 2019.

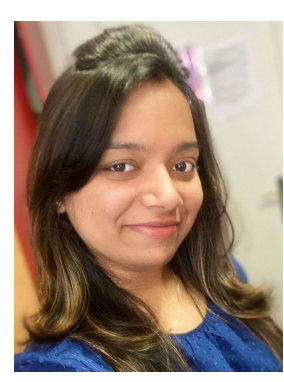

Shivani Rajendra Teli is a Ph.D. scholar in the Faculty of Electrical Engineering, Czech Technical University (CTU) in Prague. She is currently working as an Early Stage Researcher within visible light-based interoperability and networking (ViSIoN) which is a project under European Union's Horizon H2020 Marie Skłodowska-Curie Innovative Training Network (MSCA ITN). She received her M. Sc. degree from Department of Information and Communications Engineering, Pukyong National University, Busan, Korea and Bachelor's degree from Savitribai Phule Pune University, Maharashtra, India, in 2018 and 2015, respectively. Her research interests are wireless communication systems, visible light communications and optical camera communications for Internet of things. 


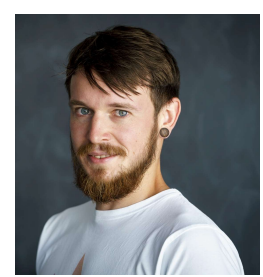

Petr Chvojka received his M.Sc. and Ph.D. degrees from the Faculty of Electrical Engineering, Czech Technical University (CTU) in Prague, in 2013 and 2018, respectively. He worked as a research fellow at the Department of Electromagnetic Field, CTU in Prague focusing on optical systems design and modelling, including inorganic and organic devices and digital signal processing techniques for visible light communications as a member of the Wireless and Fiber Optics Group. He worked at CTU in Prague until 2020 when he joined the ComAp company developing solutions for power generation and engine control.

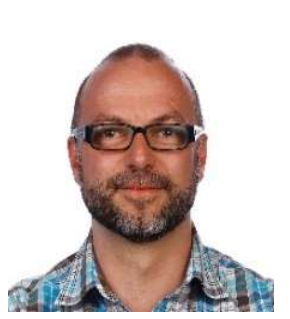

Stanislav Vítek graduated at the Czech Technical University in Prague in 2002 and received Ph.D. degree from the Czech Technical University in Prague in 2008. Recently he is an assistant professor with Dept. of Radioelectronics at the Faculty of Electrical Engineering at the Czech Technical University in Prague. Member of numerous international teams focused to networks of robotic telescopes. His main research interests are signal and image processing, embedded systems, machine learning and database systems.

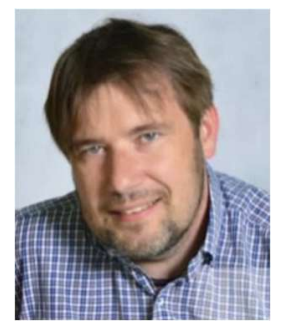

Prof. Stanislav Zvanovec received his M.Sc. and Ph.D. degrees from the Faculty of Electrical Engineering, Czech Technical University (CTU) in Prague in 2002 and 2006, respectively. To date, he works as a full professor and deputy head of the Department of Electromagnetic Field and chairperson of $\mathrm{PhD}$ branch at CTU. His current research interests include Free Space Optical and fiber optical systems, Visible Light Communications, OLED, RF over optics and electromagnetic wave propagation issues for millimeter wave band. He is the author of two books (and co-author of the recent book Visible Light Communications: Theory and Applications), several book chapters and more than 250 journal and conference papers.

Prof. Rafael Perez-Jimenez (Madrid, 1965) received his MS degree in 1991 from Universidad Politécnica de Madrid, Spain, and his Ph.D. degree (Hons) in 1995 from Universidad de Las

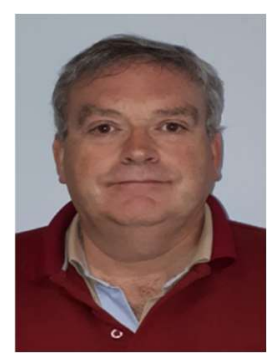

Palmas de Gran Canaria, Spain. He is a full professor at the ULPGC, where he leads the IDeTIC Research Institute. His current research interests are in the field of Optical Camera Communications (OCC), optical indoor channel characterization and the design of robust visible light communications (VLC) systems for indoor communications, especially applied for sensor interconnection and positioning. $\mathrm{He}$ is also pursuing a new $\mathrm{PhD}$ in Telecommunications History at Universidad de La Laguna. He has been awarded with the Gran Canaria Science Prize (2007), the Vodaphone Foundation Research Award (2010) and the RSEAPGC Honor Medal (2017).

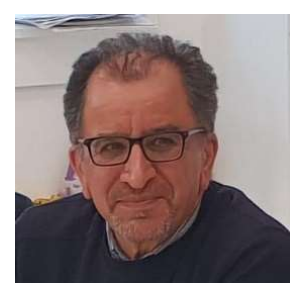

Prof. Zabih Ghassemlooy Fellow, SOA; Fellow, IET; Senior Member, IEEE, Member of ACM, CEng, BSc (Hons.) in EE Engineering, Manchester Metropolitan Univ., (1981), MSc (1984) and $\mathrm{PhD}$ (1987) from Manchester Univ., UK. $1987-88$ as a Post-Doctoral Research Fellow at City Univ., UK. 1988-2004 joined Sheffield Hallam University, UK, and 2004-14 joined Faculty of Eng. \& Env., Northumbria University, UK as an Associate Dean Research, and currently is the Head of Optical Communications Research Group. He is a Research Fellow (2016-) and a Distinguished Professor (2015-) at the Chinese Academy of Science. He was the Vice-Chair of EU Cost Action IC1101 (2011-16) and is Vice- Chair of the EU COST Action CA19111 NEWFOCUS (European Network on Future Generation Optical Wireless Communication Technologies, 2020-2024). He has published over 950 papers (more than 370 journals and 8 books), 100 keynote/invited talks, supervised 10 Research Fellows and $65 \mathrm{PhD}$ students. His research interests are in the areas of optical wireless communications, free space optics, visible light communications, hybrid RF and optical wireless communications. He is the Chief Editor of the British Journal of Applied Science and Technology and the International Journal of Optics and Applications, Associate Editor of a number of international journals, and Co-guest Editor of a number of special issues OWC. He is the Vice-Cahir of OSA Technical Group of Optics in Digital Systems (2018-). He is the Chair of the IEEE Student Branch at Northumbria University, Newcastle (2019-). From 2004-06 he was the IEEE UK/IR Communications Chapter Secretary, the Vice-Chairman (20062008), the Chairman (2008-2011), and Chairman of the IET Northumbria Network (Oct 2011-2015). 